\title{
Systematic Review of Fertility-Related Psychological Distress in Cancer Patients: informing on an improved model of care
}

\author{
Logan, S. ${ }^{\text {a, b, c }}$, Perz, J. ${ }^{\text {d, }}$ Ussher, JM ${ }^{\text {d }}$, Peate. M. ${ }^{\text {e }}$ \& Anazodo, A. ${ }^{\text {a, c, f. }}$
}

\footnotetext{
a School of Women and Children's Health, Faculty of Medicine, UNSW Australia, Sydney NSW.

${ }^{\mathrm{b}}$ Fertility \& Research Centre, Royal Hospital for Women

${ }^{c}$ Kids Cancer Centre, Sydney Children's Hospital, Sydney NSW.

${ }^{\mathrm{d}}$ Translational Health Research Institute, School of Medicine, Western Sydney University, Sydney NSW

${ }^{\mathrm{e}}$ Psychosocial Health and Wellbeing (emPoWeR) Unit, Department of Obstetrics and Gynaecology, Royal Women's Hospital, University of Melbourne, Melbourne VIC

${ }^{\mathrm{f}}$ Nelune Comprehensive Cancer Centre, Prince of Wales Hospital, Sydney NSW
}

This is the author manuscript accepted for publication and has undergone full peer review but has not been through the copyediting, typesetting, pagination and proofreading process, which may lead to differences between this version and the Version of Record. Please cite this article as doi: $10.1002 /$ pon.4927

This article is protected by copyright. All rights reserved. 
Corresponding author details: Dr Shanna Logan, School of Women and Children's Health, Faculty of Medicine, UNSW Australia, Sydney NSW. Email: shanna.logan@unsw.edu.au

This article is protected by copyright. All rights reserved. 


\begin{abstract}
Objective: Cancer patients experience reproductive concerns from diagnosis through to survivorship. However, research has yet to investigate the degree of fertility-related psychological distress at different treatment time-points; diagnosis, treatment, survivorship. Currently, cancer patients are offered fertility counselling at the time of diagnosis, to assist fertility preservation decision-making. A systematic review of the short and long term psychological impact of infertility in cancer patients would inform on an improved, longitudinal model of psychological care.
\end{abstract}

Methods: A systematic review of the literature was conducted in January 2018 utilising electronic databases Medline, EMBASE, PSYCH Info, Web of Science and SCOPUS. An initial search identified 708 potentially relevant studies. Literature was assessed that reported on fertility-related psychological distress experienced by male and female cancer patients of reproductive age ( $<45$ years) across oncology treatment time-points.

Results: A total of 47 papers were included within the final review. Fertility-related psychological distress persists from diagnosis through to survivorship, with cancer patients reporting a range of negative emotional experiences brought about by threatened infertility. In survivorship, reproductive concerns, unfulfilled desire for a child, nulliparous status and early menopause were linked to higher rates of mental health disorders and psychological distress.

Conclusions: Fertility-related psychological distress is prevalent and persistent in cancer patients and survivors. As such, patients and survivors would greatly benefit from fertilityrelated psychological support implemented into standard practice from diagnosis through to survivorship. A revised model of care is proposed.

Key words: Oncology, Cancer, Fertility, Fertility Preservation, Oncofertility, Psychological Distress, Mood Disorder, Reproductive Concerns

Word Count: 5604 
Given the improvement in life expectancy of paediatric and adult cancer patients today, preventing or reducing the risk of physical, emotional and social disruptions later in life brought about by cancer-related infertility is especially important. ${ }^{1}$ Infertility can be caused by the disease itself, or as a result from gonadal damage secondary to chemotherapy, radiotherapy, or from bone marrow transplantation. ${ }^{2,3}$ This can result in early menopause or uterine damage in women, or retrograde ejaculation and azoospermia in men, ${ }^{3}$ with survivors of childhood cancer also at risk of delayed pubertal growth. ${ }^{4}$

Cancer patients whom are of reproductive age (generally defined as those persons $<45$ years of age) may not have had the opportunity to complete their family prior to cancer diagnosis and as such may report heightened reproductive concerns. ${ }^{5,6}$ The term reproductive concerns is utilised in the literature (at times interchangeably with fertility concerns) to reflect concerns regarding a wide range of fertility factors. These may include fertility potential, pregnancy and future parenthood, recorded either by brief patient self-report ${ }^{7,8}$ or via a standardised measure. ${ }^{9}$ Reproductive concerns can persist throughout cancer treatment into survivorship and impact on areas of later life development; such as sexual health, body image or relationships ${ }^{10-12}$ and lead to lower quality of life. ${ }^{13}$ However, there is no systematic review of the literature on the psychological impact of interrupted fertility and the association between psychological distress and reproductive concerns. Yet this information would assist in informing on an improved model of psychological support for cancer patients.

Literature suggests that when cancer or cancer treatment impact on fertility potential, specialised fertility psychological interventions and counselling may be beneficial in alleviating patient's distress, assisting treatment decision-making processes and providing necessary reproductive education. ${ }^{14-17}$ Models of psychological support offer assistance at the time of diagnosis to assist in fertility preservation decision-making. ${ }^{18}$ However, psychological care does not often extend past this point, leading cancer survivors to report unmet fertility psychological care needs in survivorship. ${ }^{19,20}$

Within a cancer population, evidence suggests fertility can be related to psychological distress, ${ }^{21,22}$ a term utilised in the literature to indicate both a general negative emotional 
response, ${ }^{23}$ and a specific measurement of a mental health difficulty. ${ }^{22}$ As such, the term fertility-related psychological distress is utilised in this manuscript, to refer to any psychological distress that is either seen as a direct reaction to, or directly associated with, fertility potential, a fertility treatment, or a reproductive concern of a cancer patient. For example, both male and female patients report increased concern, worry and feelings of anxiety surrounding uncertain fertility status. ${ }^{24}$ Similarly, research indicates that cancer survivors with an unfulfilled pre-treatment desire to have a child and heightened reproductive concerns have an increased risk of depression and poorer mental health. ${ }^{21,25}$

To accurately assess the impact of fertility-related psychological distress in reproductive age cancer patients an analysis must take into account stage of cancer treatment. ${ }^{26}$ At the time of cancer diagnosis fertility interventions are likely to occur (with recommendations for fertility discussions, referral to a fertility specialist and fertility preservation) that require timely decision-making. ${ }^{27-29}$ However, as oncological treatment progresses the emotional impact of reproductive concerns may heighten. ${ }^{11}$ As such, patient's experiences of fertility-related psychological distress may alter based on stage of cancer treatment. For example, around diagnosis patients report shock, devastation, sadness and frustration at potential infertility, ${ }^{30}$, 31 elevated emotional concerns related to choice of cancer treatment and impact to future reproductive potential, ${ }^{32}$ and heightened anxiety during fertility consultations. ${ }^{33}$ Fertilityrelated psychological distress may then persist into survivorship, with research indicating persistent sadness, grief, ${ }^{34}$ anxiety, depression, panic and social isolation can remain for up to 20 years post diagnosis. ${ }^{35}$ It therefore appears important that appropriate psychological care is in place to support cancer patients throughout the entirety of their cancer journey.

Previous reviews addressing reproductive concerns or needs within a young cancer population have limited analyses to female breast cancer patients ${ }^{36,37}$ or were focused on child bearing, ${ }^{38}$ parenthood ${ }^{39}$ or the psychological impact of fertility preservation alone. ${ }^{2,40}$ These reviews have not explored fertility-related psychological distress mapped to timepoints of a cancer journey; diagnosis, treatment and survivorship; and explored how fertilityrelated psychological distress is related to reproductive concerns. Yet this knowledge is vital if we are to accurately understand the psychological impact of impaired fertility potential in 
young cancer patients across the lifespan and develop targeted psychological support. As such, the aim of this systematic review is to explore the fertility-related psychological distress and the short and long term psychological impact of interrupted fertility. This review will provide the opportunity to inform on better patient-oriented treatment and care, for cancer patients and survivors in relation to their fertility.

\section{Method}

A systematic search of online databases Medline, EMBASE, PSYCH Info, Web of Science and SCOPUS was undertaken in January 2018 using search terms (see Supplementary Table 1) to reflect cancer, psychological distress and fertility. Screening of reference lists and review articles was undertaken to accrue additional potentially relevant manuscripts. A total of 708 articles were identified via the database searches and additional manual screening after the deletion of duplicates (see Figure 1). All studies were visually screened at title and abstract level by a single reviewer, with the remaining 111 articles full text screened. A second reviewer independently read a sample of the remaining articles to determine inclusion eligibility, with any discrepancies on inclusion discussed between the two reviewers.

Inclusion criteria were met when 1) patients were of reproductive age at the time of a cancer diagnosis or during data collection (age range or mean age $\leq 45$ years) as these participants were most likely impacted by the effects of infertility; 2) data reported on fertility-related psychological distress.; 3) data was described using quantitative or qualitative methods; 4) the manuscript was in the form of a peer-reviewed journal article written in English language. Fertility-related psychological distress was quantified as either; a patient's emotional response to actual or threatened infertility; a standardised measure of fertility-related psychological distress; or a standardised measure of psychological distress that reported a direct association with a fertility treatment, fertility potential or reproductive concern. Articles reporting on sexual health outcomes, reproductive function, or reproductive concerns alone without reference to psychological distress, alongside those reporting on psychological distress not associated with fertility, were excluded from analyses. In order to accurately capture the breadth of cancer patient's experiences no limitations were placed on gender, 
cancer types or time since diagnosis. In total 47 papers describing 46 separate studies were eligible for further analysis.

The quality of final studies was assessed using the Mixed Methods Appraisal Tool (MMAT) ${ }^{41}$ a valid instrument for testing mixed method reviews. ${ }^{42}$ The MMAT assesses quality on the domains of sampling, measurements, completion of data and bias, of either qualitative or quantitative research. Scores on the MMAT vary from 25\% (one criteria met) to $100 \%$ (all criteria met). All included studies were of sound quality (75-100\%).

\section{Results}

Of the 47 final papers, 30 utilised quantitative analyses, 13 utilised qualitative techniques and 4 utilised mixed methods. Studies were published between 1999 and 2018 across eight countries, with the vast majority $(n=28)$ conducted within the United States of America. Sample sizes ranged from eight to 878 . The majority of papers reported on female patients only $(n=30)$, followed by male and female patients $(n=13)$ and male patients only $(n=4)$. Four studies investigated views of childhood cancer survivors, while a further five sampled adolescent or young adult patients ( $<26$ years). Some papers reported on one specific cancer type impacting the reproductive organs, such as breast $(n=7)$, cervical $(n=4)$, ovarian $(n=2)$, genealogical $(n=2)$ or testicular $(n=3)$ cancers; while other papers reported on a mixed sample of cancer types where cancer treatment impacted on fertility potential. Supplementary Table 2 reflects the key features and data extracted from each study on fertility-related psychological distress and full report of reproductive concerns. Separate papers reporting on the same study are combined into a single row within the data extraction table.

Only 11 of the included papers had a primary focus on the measurement of fertility-related psychological distress, with most papers primarily focusing on the experience of fertility $(n=19)$, reproductive concerns $(n=4)$, sexual function $(n=4)$ or quality of life/ general psychosocial wellbeing $(n=9)$.

Papers were divided by treatment time-point to ascertain psychological impact of infertility throughout patient's cancer journey (see Supplementary Table 3 for fertility-related psychological distress and reproductive concerns by study, across treatment time-points). 
Eight papers reported on patient experiences at the time of diagnosis, and six from the time of recent diagnosis throughout treatment. The majority of studies reported on the survivorship period; from early survivorship (approx. 2-5 years post diagnosis; $n=8$ ), survivorship generally (time frame not specified; $n=6$ ) or late survivorship (approx. 5-15 years post diagnosis; $\mathrm{n}=19)$.

\section{Diagnosis and Treatment}

\section{Fertility-Related Psychological Distress}

The risk of treatment induced infertility in newly diagnosed patients was at times as distressing and devastating to patients as the initial cancer diagnosis. ${ }^{30,43}$ Both current patients and survivors recalled feeling a range of negative emotions at the prospect of potential infertility at the time of diagnosis, including feeling scared, frightened, overwhelmed, sad, depressed, frustrated and losing control. ${ }^{30,31,43}$ Both adolescent mixed gender (11-20 years) and female breast cancer patients reported frequent worries related to fertility $(57 \%){ }^{8,44,45}$

Female patients presenting for fertility preservation treatment at the time of cancer diagnosis report poorer mental health compared to infertile non-cancer patients, including significantly greater depression (44\% vs $14 \%$ scored $>16$ on the Centre or Epidemiologic Studies Depression Scale; CES-D) and anxiety (not specific to fertility: $62 \%$ vs $27 \%$ scored $\geq 39$ on the State Trait Anxiety Inventory; STAI; $p<.05$ ), and commensurate infertility stress (on the Fertility Problem Inventory; FPI). ${ }^{46}$ Interestingly, these patients under-reported their symptoms of depression and anxiety when self-reporting (indicating 13\% and 9\% incidence of these mental health disorders respectively). Similarly, one third of female cancer patients presenting for ovarian hyper-stimulation endorsed current symptoms of depression, and anxiety that impaired functioning. ${ }^{47}$ However there was no data to indicate differences in levels of distress between those who had or had not undertaken fertility preservation at the time of diagnosis, or prevalence of mental health disorders in male patients accessing fertility preservation treatment. 
Psychological distress may persist throughout early stages of fertility and oncological treatment and then remit, opposed to reproductive concerns which remain elevated. ${ }^{32,46,48-50}$ Elevated depression (CES-D) and anxiety (STAI; p<.05) persisted throughout fertility preservation for cancer patients, despite both mood difficulties increasing in a non-cancer infertile sample. ${ }^{46}$ Elevated depression (CES-D) and fertility-related trauma (on the Impact of Event Scale (IES); a measure designed to indicate likelihood of Post-Traumatic Stress Disorder) were associated with cervical surgical treatment regardless of surgery type (radical trachelectomyy or hysterectomy); however, in both groups this distress lessened over time (both subclinical by 12 months). ${ }^{48}$ Conversely, for breast and cervical cancer patients, concern about conception, future pregnancy and menopause remained high pre and postsurgery, $(90 \%-100 \%)^{32,48}$ persisted up to two years, $(73 \%){ }^{48}$ associated with feelings of worry, anger, shock loss and fear. ${ }^{49,50}$

\section{Reproductive Concerns}

For breast cancer patients, greater reproductive concerns was associated with desire for a child in the future $(\mathrm{p}<.0001)$, number of prior pregnancies $(\mathrm{p}<.01)$ and prior difficulty conceiving, $\left(\mathrm{p}=.08\right.$, mean age 32 at diagnosis). ${ }^{8}$ Desire for a child in the future was similarly important for adolescent patients, associated with higher levels of concern and anticipated feelings of sadness, ${ }^{45}$ even though these patients reported a lack of current desire for parenthood at the time of diagnosis. ${ }^{51}$ Indeed, emotional responses to reproductive concerns may differ based on patient age; with rage and injustice reported by younger female patients (18-25 years) at thoughts of potential infertility; while future parenthood was a concern more frequently expressed by older patients (aged 26-40). ${ }^{33}$ In one sample of young ovarian cancer patients (mean age 22 years), 52\% $(n=12)$ reported fear that their disease would damage their reproductive potential; however, 39\% $(n=9)$ of this sample were not concerned about the effect of cancer treatment on their future offspring. ${ }^{52}$

Reproductive concerns were important for treatment decision-making in almost half (41\%) of one sample of newly diagnosed cervical cancer patients. ${ }^{32}$ Similarly, a large sample of breast cancer patients self-reported concern about future fertility had impacted on their oncological 
treatment decision-making ( $29 \%, \mathrm{n}=657)$; with patients more likely to report this difficulty when they also reported a desire for a future child ( $<<.0001)$, a history of severe depressive symptoms ( $\mathrm{p}=.03)$ and fertility difficulties prior to diagnosis $(\mathrm{p}=.01) .{ }^{8}$ Regret associated with fertility preservation treatment choices increased over time (12-18 months) in a second sample of breast cancer patients who had initially reported no concerns, when fertility preservation choices in early diagnosis had not been utilised. ${ }^{49}$

\section{Fertility Psychological Interventions}

Across all included studies, only three papers reported on a psychological intervention or implementation of fertility counselling/psychological support, all implemented at the time of cancer diagnosis. ${ }^{33,53,54}$ A psycho-sexual development intervention for newly diagnosed adolescent male and female patients was reported to reduce distress, emotional and sexual concerns three months post-intervention, compared to waitlist controls. ${ }^{53}$ Fertility counselling undertaken by a fertility specialist prior to cancer treatment opposed to oncologist alone was associated with better psychological health; with those who undertook counselling and proceeded with fertility preservation also reporting reduced regret, compared to those who did not proceed. ${ }^{54}$ Similarly, the presence of a psychologist was deemed a helpful opportunity to explore painful feelings and concerns surrounding infertility at the time of fertility preservation specialist consultation for female patients. ${ }^{33}$ However, this study also indicated heightened state and trait anxiety immediately post consultation (STAI state and trait $>40$ ), which further increased after patients returned home. Highlighting that exploring reproductive concerns at the time of fertility treatment decision-making was further anxiety provoking in an already anxious sample, and that patients are in need of appropriate psychological support and follow up care.

\section{Early to Late Survivorship}

\section{Fertility-Related Psychological Distress}

High rates of unknown fertility status $(77 \%),{ }^{55}$ distress, worry $(20-60 \%)^{55-59}$ and stress $(\mathrm{FPI})^{21}$ related to fertility were experienced by survivors, with distress about fertility commensurate with distress about cancer, ${ }^{60}$ and concerns reported on a regular basis (daily or 
weekly; or $25-90 \%$ of the time) ${ }^{56,57}$ When considering prevalence of fertility-related psychological distress relative to controls, research indicates that cancer survivors report greater reproductive concerns $(\mathrm{p}<.001)^{13}$ and greater distress about fertility (OR 5.23; CI 1.99-13.76, $\mathrm{n}=246)^{61}$ compared to healthy controls. However, compared to an infertile group without a history of cancer, survivors report commensurate elevated reproductive concerns (Reproductive Concerns Scale; RCS), depression (CES-D) and mental health (Quality of Life, QOL), but a lower incidence of 'moderate-severe' fertility-related trauma (IES, 30-46\% vs $59 \%, \mathrm{p}=.005){ }^{62}$

Cancer survivors experience a range of emotional responses to impacted fertility. Distress (self-reported or measured via the Brief Symptoms Inventory, BSI), ${ }^{23,24,44,53,55,60,61}$ selfreported upset, ${ }^{23,63}$ sorrow and sadness ${ }^{60}$ were reported in both male and female survivors. As were being overwhelmed or perceiving infertility to be all-consuming, ${ }^{23,56,64}$ shock, ${ }^{60}$ fear, ${ }^{60,63-66}$ anger, ${ }^{23,56,60,66,67}$ anxiety, ${ }^{24,51,60,66,68,69}$ worry, ${ }^{22,24,59,60,63,68-70}$ nervousness $^{23}$ and uncertainty. ${ }^{44,69}$

Female survivors in particular report loss of control over their reproductive future, ${ }^{13,67,70} \mathrm{a}$ feeling of time pressure in being able to form a family, ${ }^{32,63,64,68}$ feelings of grief, loss, ${ }^{24,60,64}$, $65,67,70$ devastation, ${ }^{56,65}$ and a fear of dying and leaving children without a mother. ${ }^{63}$ Female patients also reported greater distress in relation to both discussing and reminders of infertility, ${ }^{23}$ alongside greater worry about the ability to have a child and reproductive capacity, ${ }^{69}$ compared to male patients.

Findings suggest that younger patients $(<28$ years) were more concerned that cancer would interfere with fertility $(\mathrm{p}=.05)$ but were less likely to perceive themselves as infertile $(\mathrm{p}=.008)$ or have experienced difficulties with a pregnancy $(\mathrm{p}=.011),{ }^{71}$ compared to older patients $(>28$ years); with results likely due to the stage of family planning for younger patients. Similarly, in male-only samples of testicular cancer survivors, younger age was associated with greater fertility distress. ${ }^{57,58}$ One study also indicated that loneliness was anticipated by male and female adolescent patients yet to commence family planning, if their fertility was to be impaired in the future. ${ }^{23}$ 
Depression was the most commonly measured mental health disorder in survivors, with approximately $22-30 \%$ of female (post-menopausal, infertile or pregnant at cancer diagnosis) or male survivors (with mixed fertility status), reporting clinically significant depression (not specific to fertility). ${ }^{25,62,66,72-76}$ One study reported clinically significant distress about fertility on the BSI (20\%) was mainly accounted for by elevated anxiety, opposed to depression in a female sample. ${ }^{77}$ However, there was no further information on the prevalence of clinically heightened anxiety in male or female cancer survivors, despite feelings of anxiety and worry being frequently reported by this cohort. Measures of fertilityrelated trauma (IES) indicate $20 \%-72 \%$ of female survivors experience clinically significant distress; ${ }^{22,62,73,77}$ in particular experiencing intrusive memories, ${ }^{77}$ and there was a strong positive association between heightened trauma (IES) and depression (CES-D; $<<.0001){ }^{73}$ No studies reported on the prevalence of trauma in male-only samples.

Emotional wellbeing, opposed to psychological distress, appears to be a separate construct and not simply the inverse of emotional ill health. For example, greater emotional wellbeing was linked to those cancer patients whose fertility had not been impeded $;^{13}$ however, it was not associated with fertility impacting cancer treatment, patient perceptions of infertility or desiring a future child. ${ }^{71}$

\section{Reproductive Concerns}

Patients report both constant and persistent reproductive concerns into survivorship ${ }^{49,63}$ that disrupt the life narrative; with a realisation that cancer would affect them for the rest of their lives ${ }^{23}$ leading to a future with no meaning or purpose, ${ }^{56,66}$ lack of normalcy and a feeling of being 'damaged goods', inadequate or that their body was 'disgusting', or simply affected feelings about their self $(25-27 \%) .{ }^{44,60,65,66,68}$ Uncertainty regarding fertility status, loss of control of reproductive status, reminders of infertility or accepting infertility status being particularly emotionally difficult for patients. ${ }^{13,23,56}$ It appears that reproductive concerns are independent from actual ability to have children, with male testicular cancer patients having higher levels of self-reported fertility distress and also perceiving greater difficulty in 
fathering children, compared with healthy controls, despite being more likely to have fathered children. ${ }^{61}$

Reproductive concerns were associated with depression (not specific to fertility: $\mathrm{p}<.0001$ $\mathrm{p}=.0002),{ }^{75,76}$ with odds of 'moderate' to 'severe' depression 1.3 times higher with every five unit increase on the Reproductive Concerns After Cancer scale (RCAC) ${ }^{76}$ alongside poorer mental health (QOL, $\mathrm{p}<.001)$, more cancer specific distress $(\mathrm{p}<.001)$ and significantly poorer quality of life. ${ }^{13}$ It therefore appears likely that for cancer survivors who report persistent and heightened reproductive concerns in survivorship, their likelihood for development of a mental health disorder is increased.

Unfulfilled desire for a child and interrupted childbearing (reported in $17 \%$ of male and $30 \%$ of female survivors) increase the risk for poorer mental health (not specific to fertility: $\mathrm{p}=.024),{ }^{21}$ more fertility-related trauma symptoms (IES intrusion and avoidance subscales, $\mathrm{p}<.001)$, higher reproductive concerns $(\mathrm{RCS}, \mathrm{p}<.001) ;{ }^{22,75}$ greater cancer distress $(\mathrm{p}<.01)$ and lower psychological wellbeing (not specific to fertility: $\mathrm{p}<.001$ ) ${ }^{13}$ Approximately $50 \%$ of survivors with a pre-treatment desire for a child report persistent unfulfilled desires, and this cohort tended to be younger age and nulliparous. ${ }^{21}$ Similarly, unfulfilled desire for a child in those who are nulliparous is also associated with depression (not specific to fertility: CES-D; $\mathrm{p}=.03)^{75}$

Childless status is also important; associated with greater distress about fertility in male survivors, ${ }^{57,58}$ and greater fertility-related trauma (IES, $\mathrm{p}<.01$ ) and reproductive concerns (RCS, $\mathrm{p}<.001)$ in female survivors with primary $(\mathrm{n}=38)$ opposed to secondary infertility $(n=39) .{ }^{22}$ Similarly, depression (not specific to fertility: CES-D) was associated with those who had no children prior to a cancer diagnosis $(\mathrm{p}=.004)^{73,75}$ and greater cancer-related trauma reported in those who had been advised to terminate a pregnancy at diagnosis (IES; $\mathrm{p}=.02) .{ }^{77}$ Conversely, having children appears to be protective, with women scoring lower in depression (not specific to fertility: CES-D) if they had children $(P=0.047)^{73}$, had one or more live births $(\mathrm{p}=.0007)$ or attempted a pregnancy compared to avoiding pregnancy after cancer $(\mathrm{p}=.004){ }^{75}$ 
Risk of depression in post-menopausal survivors (opposed to those with ovarian function) may be up to 3.4 times $(\mathrm{p}<.001-\mathrm{p}=.02){ }^{25,75} \mathrm{Up}$ to one third of female survivors reported being bothered by menopausal symptoms, ${ }^{73}$ causing fear and anxiety; ${ }^{66}$ with greater impact ('bother') of menopausal symptoms associated with higher cancer-related trauma and depression (IES, $\mathrm{p}<.001$; CES-D, $\mathrm{p}<.0001-.001) .{ }^{73}$ Another study reported a weak association between poorer mental health (Mental Component Summary Scale, MCS; p=.10) in young female breast cancer patients (24-34 years) who had experienced early menopause post oncological treatment compared to those whom had not, and no significant association in older patients (34-51 years) ${ }^{34}$ suggesting younger patients may be more emotionally impacted by early menopause. Sexual dysfunction in young adult male and female survivors also indicated a significantly greater risk of depression, anxiety disorders ( $>13$ years at diagnosis, mean age 27 years at time of study; score of $\geq 57$ on the BSI) ${ }^{72}$ or self-reported distress (18-31 years at time of study). ${ }^{24}$

\section{Oncofertility Treatment and Care}

Cancer site and treatment type influenced experience of fertility-related psychological distress. In male and female cancer survivors, a cancer site close to reproductive organs was associated with heightened anxiety, ${ }^{51}$ while having chemotherapy or surgery was related to elevated distress about fertility in male patients, compared to those who had not. ${ }^{61}$ Similarly, greater symptoms of depression were reported in irradiated compared to cervical cancer patients treated with surgery or healthy controls. ${ }^{74}$

Patient's experience with oncofertility care was also important in the expression of psychological distress. Lack of fertility support, patient knowledge or provision of fertility information was associated with experiences of uncertainty and concern, alongside higher levels of depression (not specific to fertility: CES-D) and cancer or fertility-related trauma (IES, $\mathrm{p}<.01-\mathrm{p}=.02)^{60,62,69,77}$ Discussing fertility with clinicians or family ${ }^{23,30}$ brought about negative emotional responses when patients felt fertility was not given full consideration. ${ }^{51}$ Feelings of anxiety and fear also led to avoidance of ongoing fertility testing in male and female childhood cancer survivors. ${ }^{68}$ 


\section{Discussion}

This manuscript sought to systematically review the literature on fertility-related psychological distress reported by cancer patients of reproductive age across treatment timepoints, in order to understand the psychological impact of actual or threatened infertility. Results suggest that heightened fertility-related psychological distress and poorer psychological health are both prevalent and persistent within this population, and as such it is worthwhile considering an improved model of psychological care.

Reproductive concerns persist throughout diagnosis, oncological treatment and into survivorship, with survivors reporting a range of negative emotional responses to actual or threatened infertility. Impaired fertility can be linked to an intrinsic sense of self and may interrupt the life narrative. Interestingly, reproductive concerns may persist despite no reported physical difficulties in reproductive ability, indicating that perception of self as fertile may be important in mediating reproductive concerns or distress. It would therefore be beneficial for clinicians to explore with patients not only their reproductive function, but the thoughts and feelings patients have surrounding their fertility potential and the impact this has to their quality of life throughout the cancer journey, in order to better capture and address these concerns.

Clinically elevated rates of depression, anxiety and trauma were noted within a cancer population at the time of diagnosis when accessing fertility preservation treatment. Initial heightened psychological distress may present as a reaction to the cancer diagnosis; however, given heightened distress persists throughout fertility treatment, and anxiety may elevate after discussing fertility within consultations, it is paramount that cancer patients have the opportunity to access specialised psychological support within their fertility-related care at this treatment time point. Data indicates a lessening of psychological distress throughout oncological treatment, with the cessation of clinically elevated trauma and depression. ${ }^{48}$

In survivorship, both male and female cancer survivors with impacted fertility present with an increased risk for significant psychological distress, commensurate with a mental health disorder diagnosis. Specifically, findings indicate an increased prevalence of clinically 
significant depression and heightened risk for anxiety. Currently, there is no data on prevalence of fertility-related trauma in male patients at any stage of cancer treatment; however, results in female samples indicate prevalence is very high, up to $72 \%{ }^{73}$ There appear to be particular risk factors for the later expression of significant psychological distress; with heightened reproductive concerns, unfulfilled desire for a child, nulliparous status, menopausal status and sexual dysfunction all linked with poorer mental health outcomes.

Of particular note, the reproductive concern of unfulfilled desire to have a child in female patients was linked to significantly more trauma, depression, poorer mental health, higher reproductive concerns and lower sexual satisfaction, compared to those without interrupted childbearing; with greatest distress amongst those who had no children prior to a cancer diagnosis. This finding is particularly important within a reproductive-aged cancer population who are unlikely to have had the opportunity to complete their family prior to a cancer diagnosis. Indeed, greater disruption to family planning by infertility may also account for why younger cohorts within a reproductive age bracket experience greater distress. It is therefore important that clinicians assess desire for a child/more children within survivorship, alongside biological reproductive symptoms, to ensure patients are able to access necessary fertility counselling and psychological support.

The results of this systematic review suggest that it is vital that both male and female cancer patients of a reproductive age have access to fertility-related psychological care if desired. Limited data available suggests the implementation of fertility interventions in psychological support at diagnosis are beneficial for patient's mental health, ${ }^{33,53,54}$ and lack of support or information throughout survivorship is detrimental. ${ }^{60,62,69,77}$ Psychological care that is positioned throughout the cancer journey may better manage persistent fertility-related psychological distress and the ongoing uncertainty and distress associated with actual or threatened impaired fertility. This view is consistent with previous research, stating both the perceived patient benefit and necessity of psychological services within oncofertility care. ${ }^{14}$, 17 


\section{Limitations}

Although this review captures a wide range of studies, the majority did not focus exclusively on fertility-related psychological distress or reproductive concerns. In the majority of cases each study reports a small quantity of data which was captured as part of a broader analysis. As such, there is the risk that with this less focused approach in the reported studies, vital information has not been captured. Additionally, by capturing those patient samples with a mean or range of less than 45 years of age, there is the potential that articles describing the longer term fertility-related psychological impact of adult survivors were missed. Within future research it may be worthwhile further exploring how this distress persists post reproductive age or the impact to parents/partners within the larger family system.

Grouping all cancer types means variable information is lost, and there is a bias in the literature with a large proportion of fertility research informing on breast or lymphoma cancers, or female patients only. Moreover, a focus on the psychological care needs of cancer patients has biased the review to focus on the negative emotional experiences of cancer patients in relation to their fertility, and as such literature on the positive psychological aspects of fertility after cancer have not been captured within the literature search. For example, recent research indicates that the majority of recently diagnosed melanoma female patients of reproductive age report no concerns about future family planning. ${ }^{78}$

However, this lens was necessary to inform on the level of support needed for those cancer patients who do report fertility-related psychological distress throughout treatment and survivorship, and to inform on a model of care that may be beneficial for all cancer patients who express a need for additional support. The limited number of interventions, although shown to be beneficial, do limit the ability to draw definitive conclusions about how services may be improved to better incorporate psychological care, or to determine clear pathways and/ or processes by which improvements may be implemented. As such, future research that investigates the influence of additional psychological support throughout cancer treatment and survivorship is needed.

\section{Clinical Implications}


Based on the results of this review a number of treatment recommendations can be suggested in order to better support patients with their psychological health within oncofertility care. If fertility-related psychological distress persists into survivorship, it is worthwhile considering the application of psychological interventions throughout the entire cancer journey as an improved model of psychological care. It is recommended that the provision of specialised mental health treatment be available to those patients that report significant levels of distress. This psychological specialised assistance may be integrated into current fertility counselling practices, or may need to be accessed as an additional service, if beyond the scope of training of a fertility counselling clinician. Therefore, fertility treating centres should ensure they are able to access or refer to appropriate psychological services as part of fertility-related care.

It is imperative that patients have ongoing access to fertility services, such as a fertility specialist and fertility preservation. Access to fertility preservation would serve to reduce physical reproductive barriers to later family planning and may assist with the significant distress incurred by those cancer survivors who have not yet had an opportunity to complete their family. Given the provision of fertility information and increased fertility knowledge reduce psychological distress ${ }^{11}$ and are important to those with heightened anxiety, ${ }^{79}$ the use of fertility preservation decision aids that assist cancer patients in making fertility preservation treatment choices is recommended, to complement these services.

It is also recommended that ongoing communication about fertility and general supportive care be offered to all patients throughout all stages of cancer treatment. The provision of an ongoing platform for patients to discuss their fertility psychological needs within oncofertility will allow patients to be better supported emotionally, allow clinicians to link patients with specialist services in a timely manner and thereby reduce the likelihood for the expression of significant mental health difficulties due to infertility.

\section{Conclusions}

The interruption to fertility brought about by cancer and cancer treatment is inextricably linked to indicators of emotional ill health. Fertility-related psychological distress is prevalent and persistent within a cancer patient population diagnosed at reproductive age. This distress 
and concern is not only related to later reproductive function, but the patient's perception of fertility status, and impacts on psychological functioning well into survivorship.

Improvements in psychological support within oncofertility care are therefore not only beneficial but imperative, to reduce suffering at the time of diagnosis and into survivorship. When the provision of cancer treatment impacts fertility potential there is a duty of care by clinicians to adequately support cancer patients both medically and psychologically. Engagement with this care early in a patient's cancer journey will assist in the ongoing facilitation of psychological support as warranted throughout the cancer journey. When patients report with significant clinically elevated levels of psychological distress it is important that they have access to specialised psychological services.

Ongoing fertility-related supportive care, such as via the provision of fertility information, discussions of fertility with patients, provision of counselling or fertility-related education, may all positively influence or mediate the level of fertility-related psychological distress that patient's experience. The provision of oncofertility care that is grounded in psychologically supportive practice will benefit patients greatly, in being able to respond to and cope with the psychological stressors inherent in interrupted fertility.

Funding Source: This work is supported by Kids Cancer Alliance (KCA), a CINSW Translational Cancer Research Centre (15/TRC/1-04). 


\section{References}

1. Ganz PA, Bower JE and Stanton AL. Special Issues in Younger Women with Breast Cancer. In: Ganz PA, (ed.). Improving Outcomes for Breast Cancer Survivors: Perspectives on Research Challenges and Opportunities. Berlin: Springer-Verlag Berlin, 2015, p. 9-21.

2. Tschudin S and Bitzer J. Psychological aspects of fertility preservation in men and women affected by cancer and other life-threatening diseases. Hum Reprod Update. 2009; 15: 587-97.

3. Nieman $\mathrm{CL}$, Kazer R, Brannigan RE, et al. Cancer survivors and infertility: a review of a new problem and novel answers. J Support Oncol. 2006; 4: 171-8.

4. Pacey AA. Fertility issues in survivors from adolescent cancers. Cancer Treat Rev. 2007; 33: 646-55.

5. Gershenson DM, Miller AM, Champion VL, et al. Reproductive and sexual function after platinum-based chemotherapy in long-term ovarian germ cell tumor survivors: A gynecologic oncology group study. J Clin Oncol. 2007; 25: 2792-7.

6. Ruddy KJ, Gelber S, Ginsburg ES, et al. Menopausal symptoms and fertility concerns in premenopausal breast cancer survivors: a comparison to age- and gravidity-matched controls. Menopause. 2011; 18: 105-8.

7. Benedict C, Shuk E and Ford JS. Fertility Issues in Adolescent and Young Adult Cancer Survivors. J Adolesc Young Adult Oncol. 2016; 5: 48-57.

8. Partridge AH, Gelber S, Peppercorn J, et al. Web-based survey of fertility issues in young women with breast cancer. J Clin Oncol. 2004; 22: 4174-83.

9. Gorman JR, Su HI, Pierce JP, Roberts SC, Dominick SA and Malcarne VL. A multidimensional scale to measure the reproductive concerns of young adult female cancer survivors. Journal of cancer survivorship : research and practice. 2014; 8: 218-28.

10. Incrocci L, Hop WCJ, Wijnmaalen A and Slob AK. Treatment outcome, body image, and sexual functioning after orchiectomy and radiotherapy for Stage I-II testicular seminoma.

International Journal of Radiation Oncology Biology Physics. 2002; 53: 1165-73.

11. Carter J, Raviv L, Sonoda Y, Chi DS and Abu-Rustum NR. Recovery Issues of FertilityPreserving Surgery in Patients With Early-Stage Cervical Cancer and a Model for Survivorship The Physician Checklist. International Journal of Gynecological Cancer. 2011; 21: 106-16.

12. Lehmann $V$, Gronqvist $H$, Engvall $G$, et al. Negative and positive consequences of adolescent cancer 10 years after diagnosis: an interview-based longitudinal study in Sweden. Psychooncology. 2014; 23: 1229-35.

13. Wenzel L, Dogan-Ates A, Habbal R, et al. Defining and Measuring Reproductive Concerns of Female Cancer Survivors. Journal of the National Cancer Institute Monograph. 2005; 34: 94-8.

14. Besse $D$, Bellavia $M$, de Ziegler $D$ and Wunder $D$. Fertility and cancer: psychological support in young women who contemplate emergency assisted reproductive technologies (ART) prior to chemo- and/or radiation-therapy. Swiss Med Wkly. 2010; 140: w13075.

15. Benedict $C$, Thom B and Kelvin JF. Young adult female cancer survivors' decision regret about fertility preservation. J Adolesc Young Adult Oncol. 2015; 4: 213-8.

16. Chan JL, Letourneau J, Salem W, et al. Regret around fertility choices is decreased with pretreatment counseling in gynecologic cancer patients. Journal of cancer survivorship : research and practice. 2017; 11: 58-63.

17. Canavarro MC and Pires RSA. The impact of gynecological cancer on reproductive issues and pregnancy: Psychological implications. Current Women's Health Reviews. 2011; 7: 367-78. 
18. Bradford A and Woodard TL. Novel Psychological Intervention for Decision Support in Women Considering Fertility Preservation Before Cancer Treatment. J Adolesc Young Adult Oncol. 2017; 6: 348-52.

19. Geue K, Richter D, Schmidt R, et al. The Desire for Children and Fertility Issues Among Young German Cancer Survivors. Journal of Adolescent Health. 2014; 54: 527-35.

20. Miedema B, Easley J and Robinson LM. Do current cancer follow-up care practices meet the needs of young adult cancer survivors in Canada? A qualitative inquiry. Curr Oncol. 2013; 20: 14-22. 21. Armuand GM, Wettergren L, Rodriguez-Wallberg KA and Lampic C. Desire for children, difficulties achieving a pregnancy, and infertility distress 3 to 7 years after cancer diagnosis. Support Care Cancer. 2014; 22: 2805-12.

22. Canada AL and Schover LR. The psychosocial impact of interrupted childbearing in long-term female cancer survivors. Psychooncology. 2012; 21: 134-43.

23. Benedict C, Shuk E and Ford JS. Fertility Issues in Adolescent and Young Adult Cancer Survivors. J Adolesc Young Adult Oncol. 2016; 5: 48-57.

24. Frederick NN, Recklitis CJ, Blackmon JE and Bober S. Sexual Dysfunction in Young Adult Survivors of Childhood Cancer. Pediatric Blood and Cancer. 2016; 63: 1622-8.

25. Bisseling KCHM, Kondalsamy-Chennakesavan S, Bekkers RLM, Janda M and Obermair A. Depression, anxiety and body image after treatment for invasive stage one epithelial ovarian cancer. Australian and New Zealand Journal of Obstetrics and Gynaecology. 2009; 49: 660-6.

26. Knapp CA, Quinn GP and Murphy D. Assessing the Reproductive Concerns of Children and Adolescents with Cancer: Challenges and Potential Solutions. J Adolesc Young Adult Oncol. 2011; 1: 31-5.

27. NCNN Clinical Practice Guidelines in Oncology for Adolescent and Young Adult (AYA) Oncology (Version I.2012). In: National Comprehensive Cancer Network I, (ed.). Fort Washington, PA2011.

28. Fertility: Assessment and treatment for people with fertility problems (NICE clinical guideline CG156). In: Excellence NIfHaC, (ed.). London2013.

29. Fertility preservation for AYAs diagnosed with cancer: Guidance for health professionals. In: AYA cancer fertility preservation guidance working group COSoA, (ed.). Sydney: Cancer Council Australia, 2014.

30. Armuand GM, Wettergren L, Rodriguez-Wallberg KA and Lampic C. Women more vulnerable than men when facing risk for treatment-induced infertility: A qualitative study of young adults newly diagnosed with cancer. Acta Oncologica. 2015; 54: 243-52.

31. Keim-Malpass J, Baernholdt M, Erickson JM, Ropka ME, Schroen AT and Steeves RH. Blogging Through Cancer Young Women's Persistent Problems Shared Online. Cancer Nurs. 2013; 36: 163-72.

32. Carter J, Sonoda Y and Abu-Rustum NR. Reproductive concerns of women treated with radical trachelectomy for cervical cancer. Gynecologic Oncology. 2007; 105: 13-6.

33. Razzano A, Revelli A, Delle Piane L, et al. Fertility preservation program before ovarotoxic oncostatic treatments: role of the psychological support in managing emotional aspects. Gynecol Endocrinol. 2014; 30: 822-4.

34. Ganz PA, Greendale GA, Peterson L, Kahn B and Bower JE. Breast Cancer in Younger Women: Reproductive and late health effects of treatment. J Clin Oncol. 2003; 21: 4184-93.

35. Dizon DS. Quality of Life after Breast Cancer: Survivorship and Sexuality. Breast Journal. 2009; 15: 500-4. 
36. Peate $M$, Meiser $B$, Hickey $M$ and Friedlander $M$. The fertility-related concerns, needs and preferences of younger women with breast cancer: a systematic review. Breast Cancer Research and Treatment. 2009; 116: 215-23.

37. Howard-Anderson J, Ganz PA, Bower JE and Stanton AL. Quality of life, fertility concerns, and behavioral health outcomes in younger breast cancer survivors: A systematic review. J Nat/ Cancer Inst. 2012; 104: 386-405.

38. Gonçalves V, Sehovic I and Quinn G. Childbearing attitudes and decisions of young breast cancer survivors: a systematic review. Hum Reprod Update. 2014; 20: 279-92.

39. Sobota A and Ozakinci G. Fertility and parenthood issues in young female cancer patients-a systematic review. J. 2014; 8: 707-21.

40. Deshpande NA, Braun IM and Meyer FL. Impact of fertility preservation counseling and treatment on psychological outcomes among women with cancer: A systematic review. Cancer. 2015; 121: 3938-47.

41. Pluye P, Gagons M-P, Griffiths F and Johnson-Lafleur J. A Scoring System for Appraising Mixed Methods Research , and Concomitantly Appraising Qualitative, Quantitative and Mixed Methods Primary Studies in Mixed Studies Reviews. International Journal of Nursing Studies. 2009; 46: 529-46.

42. Pace R, Pluye $P$, Bartlett $\mathrm{G}$, et al. Testing the Reliability and Efficacy of the Pilot Mixed Method Appraisal Tool (MMAT) for Systematic Mixed Studies Review. International Journal of Nursing Studies. 2012; 49: 47-53.

43. Yee $\mathrm{S}$, Abrol K, McDonald M, Tonelli M and Liu KE. Addressing oncofertility needs: Views of female cancer patients in fertility preservation. Journal of Psychosocial Oncology. 2012; 30: 331-46. 44. Crawshaw MA and Sloper P. 'Swimming against the tide' - the influence of fertility matters on the transition to adulthood or survivorship following adolescent cancer. Eur J Cancer Care. 2010; 19: 610-20.

45. Quinn GP, Knapp C, Murphy D, Sawczyn K and Sender L. Congruence of reproductive concerns among adolescents with cancer and parents: pilot testing an adapted instrument. Pediatrics. 2012; 129: e930-6.

46. Lawson AK, Klock SC, Pavone ME, Hirshfeld-Cytron J, Smith KN and Kazer RR. Prospective study of depression and anxiety in female fertility preservation and infertility patients. Fertility and Sterility. 2014; 102: 1377-84.

47. Lawson AK, Klock SC, Pavone ME, Hirshfeld-Cytron J, Smith KN and Kazer RR. Psychological Counseling of Female Fertility Preservation Patients. Journal of Psychosocial Oncology. 2015; 33: 333-53.

48. Carter J, Sonoda Y, Baser RE, et al. A 2-year prospective study assessing the emotional, sexual, and quality of life concerns of women undergoing radical trachelectomy versus radical hysterectomy for treatment of early-stage cervical cancer. Gynecologic Oncology. 2010; 119: 358-65. 49. Connell S, Patterson C and Newman B. A Qualitative Analysis of Reproductive Issues Raised by Young Australian Women with Breast Cancer. Health Care for Women International. 2006; 27: 94110.

50. Avis NE, Crawford S and Manuel J. Psychosocial problems among younger women with breast cancer. Psychooncology. 2004; 13: 295-308.

51. Crawshaw MA, Glaser AW, Hale JP and Sloper P. Male and female experiences of having fertility matters raised alongside a cancer diagnosis during the teenage and young adult years. Eur J Cancer Care. 2009; 18: 381-90. 
52. Sait KH. Conservative treatment of ovarian cancer safety, ovarian function preservation, reproductive ability, and emotional attitude of the patients in Saudi Arabia. Saudi Medical Journal. 2011; 32: 913-8.

53. Canada AL, Schover LR and Li YS. A pilot intervention to enhance psychosexual development in adolescents and young adults with cancer. Pediatr Blood Cancer. 2007; 49: 824-8.

54. Letourneau JM, Ebbel EE, Katz PP, et al. Pretreatment fertility counseling and fertility preservation improve quality of life in reproductive age women with cancer. Cancer. 2013; 118: 1710-7.

55. Lehmann V, Keim MC, Nahata L, et al. Fertility-related knowledge and reproductive goals in childhood cancer survivors: short communication. Human Reproduction. 2017; 32: 2250-3.

56. Carter J, Rowland K, Chi D, et al. Gynecologic cancer treatment and the impact of cancerrelated infertility. Gynecologic Oncology. 2005; 97: 90-5.

57. Rieker PP, Fitzgerald EM and Kalish LA. Adaptive behavioral responses to potential infertility among survivors of testis cancer. J Clin Oncol. 1990; 8: 347-55.

58. Rieker PP, Fitzgerald EM, Kalish LA, et al. Psychosocial factors, curative therapies, and behavioral outcomes. A comparison of testis cancer survivors and a control group of healthy men. Cancer. 1989; 64: 2399-407.

59. Saito K, Suzuki K, Iwasaki A, Yumura Y and Kubota Y. Sperm cryopreservation before cancer chemotherapy helps in the emotional battle against cancer. Cancer. 2005; 104: 521-4.

60. Ussher JM, Perz J and (ACFST) TACaFST. Threat of biographical disruption: the gendered construction and experience of infertility following cancer for women and men. BMC Cancer. 2018; 18: 1-17.

61. Kim C, McGlynn KA, McCorkle R, et al. Fertility among testicular cancer survivors: a casecontrol study in the US. J Cancer Surviv-Res Pract. 2010; 4: 266-73.

62. Carter J, Raviv L, Applegarth L, et al. A cross-sectional study of the psychosexual impact of cancer-related infertility in women: third-party reproductive assistance. J. 2010; 4: 236-46.

63. Ruddy KJ, Greaney ML, Sprunck-Harrild K, Meyer ME, Emmons KM and Partridge AH. Young Women with Breast Cancer: A Focus Group Study of Unmet Needs. J Adolesc Young Adult Oncol. 2013; 2: 153-60.

64. Lloyd PA, Briggs EV, Kane N, Jeyarajah AR and Shepherd JH. Women's experiences after a radical vaginal trachelectomy for early stage cervical cancer. A descriptive phenomenological study. Eur J Oncol Nurs. 2014; 18: 362-71.

65. Dryden A, Ussher JM and Perz J. Young women's construction of their post-cancer fertility. Psychol Health. 2014; 29: 1341-60.

66. Parton C, Ussher JM and Perz J. Experiencing menopause in the context of cancer: Women's constructions of gendered subjectivities. Psychol Health. 2017; 32: 1109-26.

67. Wenzel L, Berkowitz RS, Newlands E, et al. Quality of life after gestational trophoblastic disease. J Reprod Med. 2002; 47: 387-94.

68. Nilsson J, Jervaeus A, Lampic C, et al. 'Will I be able to have a baby?' Results from online focus group discussions with childhood cancer survivors in Sweden. Human Reproduction. 2014; 29 : 2704-11.

69. Yi J, Kim MA and Sang J. Worries of childhood cancer survivors in young adulthood. Eur J Oncol Nurs. 2016; 21: 113-9.

70. Dunn J and Steginga SK. Young women's experience of breast cancer: defining young and identifying concerns. Psychooncology. 2000; 9: 137-46. 
71. Schover LR, Rybicki LA, Martin BA and Bringelsen KA. Having children after cancer - A pilot survey of survivors' attitudes and experiences. Cancer. 1999; 86: 697-709.

72. Bober SL, Zhou ES, Chen B, Manley PE, Kenney LB and Recklitis CJ. Sexual Function in Childhood Cancer Survivors: A Report from Project REACH. J Sex Med. 2013; 10: 2084-93.

73. Carter J, Chi DS, Brown CL, et al. Cancer-related infertility in survivorship. International Journal of Gynecological Cancer. 2010; 20: 2-8.

74. Frumovitz $M$, Sun CC, Schover L, et al. Quality of life and sexual functioning in cervical cancer survivors. J Clin Oncol. 2005; 23: 7428-36.

75. Gorman JR, Malcarne VL, Roesch SC, Madlensky L and Pierce JP. Depressive symptoms among young breast cancer survivors: the importance of reproductive concerns. Breast Cancer Research and Treatment. 2010; 123: 477-85.

76. Gorman JR, Su HI, Roberts SC, Dominick SA and Malcarne VL. Experiencing reproductive concerns as a female cancer survivor is associated with depression. Cancer. 2015; 121: 935-42.

77. Henry M, Huang LN, Sproule BJ and Cardonick EH. The psychological impact of a cancer diagnosed during pregnancy: determinants of long-term distress. Psychooncology. 2012; 21: 444-50. 78. Atkinson TM, Noce NS, Hay J, Rafferty BT and Brady MS. Illness-Related Distress in Women with Clinically Localized Cutaneous Melanoma. Ann Surg Oncol. 2013; 20: 675-9.

79. Nakayama K, Liu P, Detry M, et al. Receiving information on fertility- and menopause-related treatment effects among women who undergo hematopoietic stem cell transplantation: changes in perceived importance over time. Biol Blood Marrow Transplant. 2009; 15: 1465-74. 
Figure 1: Flowchart of inclusion/exclusion process
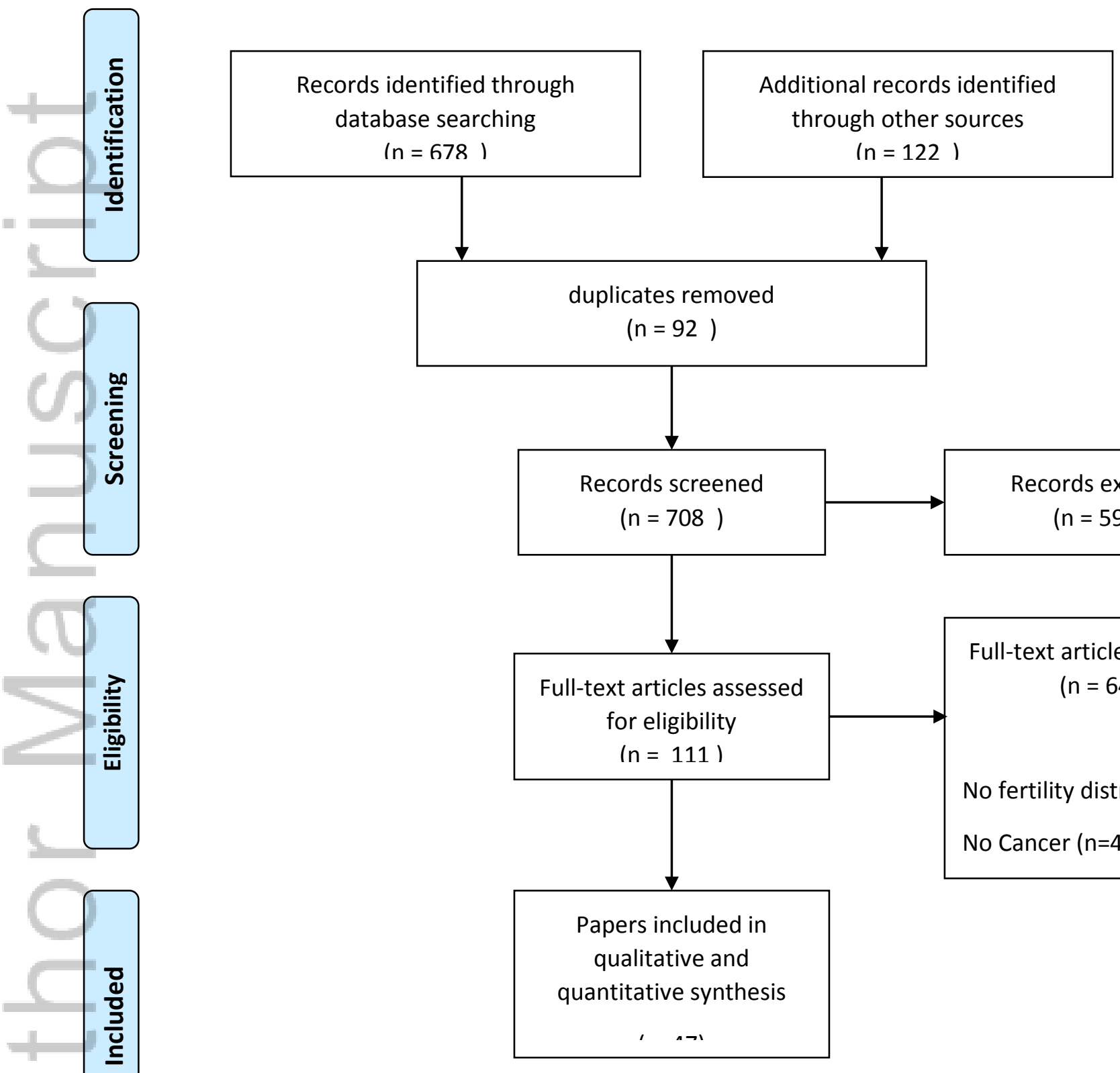

duplicates removed

$(n=92)$

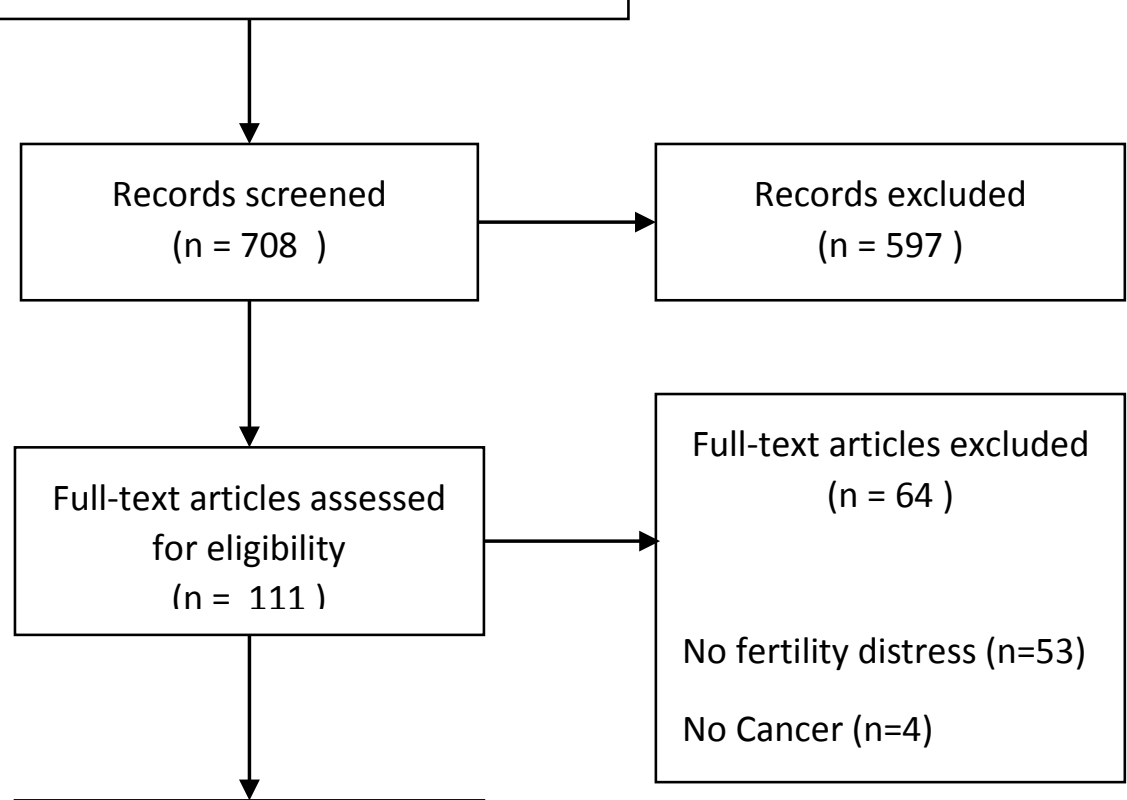

Papers included in

qualitative and

quantitative synthesis

This article is protected by copyright. All rights reserved. 
Figure 1: Flowchart of inclusion/exclusion process
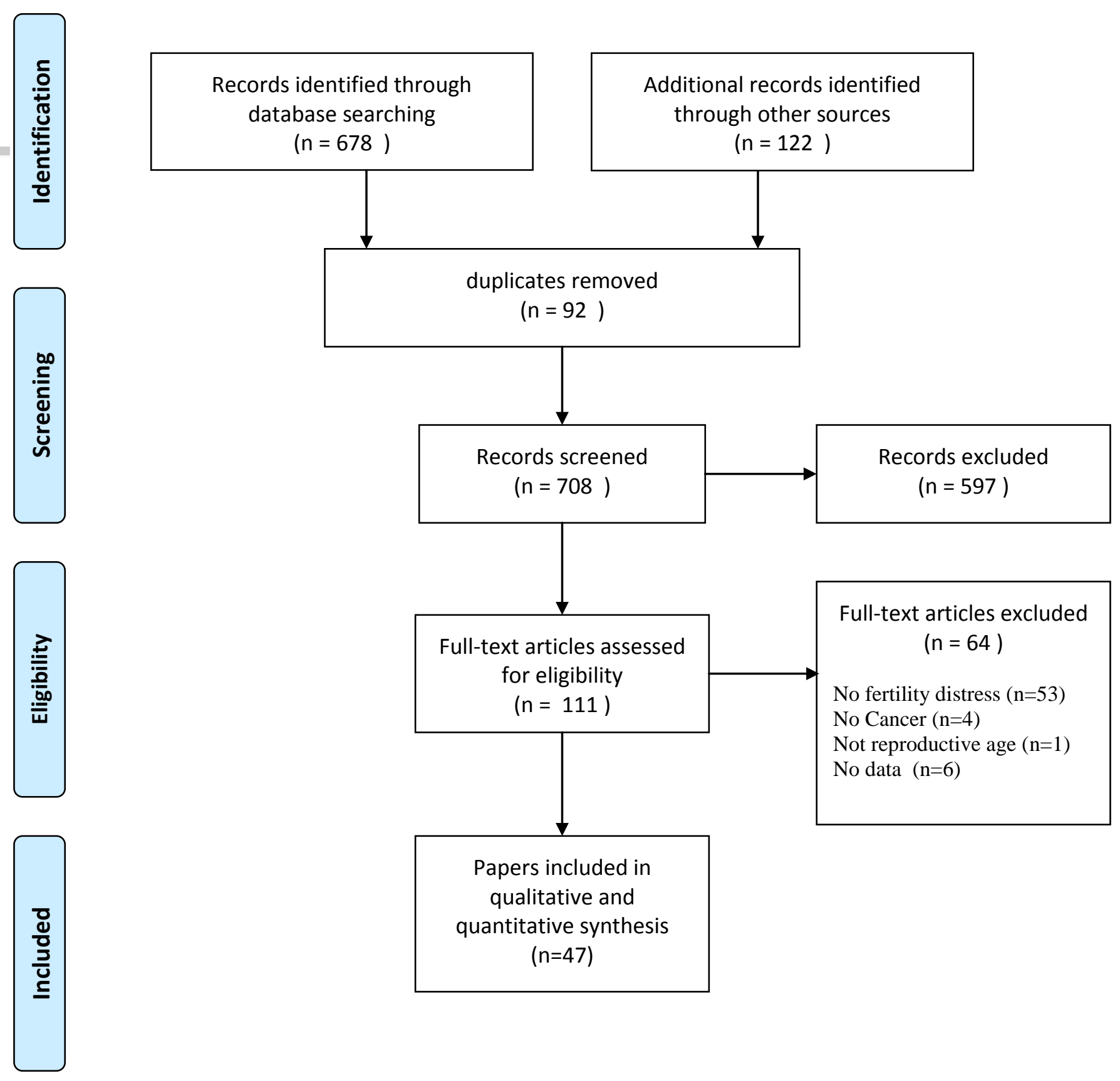

This article is protected by copyright. All rights reserved. 


\section{University Library}

\section{- M M N E R VA A gateway to Melbourne's research publications}

Minerva Access is the Institutional Repository of The University of Melbourne

Author/s:

Logan, S;Perz, J;Ussher, JM;Peate, M;Anazodo, A

Title:

Systematic review of fertility-related psychological distress in cancer patients: Informing on an improved model of care

Date:

2019-01-01

\section{Citation:}

Logan, S., Perz, J., Ussher, J. M., Peate, M. \& Anazodo, A. (2019). Systematic review of fertility-related psychological distress in cancer patients: Informing on an improved model of care. PSYCHO-ONCOLOGY, 28 (1), pp.22-30. https://doi.org/10.1002/pon.4927.

Persistent Link:

http://hdl.handle.net/11343/284784 